\title{
Verfassungshüter ohne politischen Rückhalt. Das tschechoslowakische Verfassungsgericht nach 1920 im Vergleich mit Österreich*
}

In Österreich und in der Tschechoslowakei wurde im Jahr 1920 ein neuartiges Modell der Verfassungsgerichtsbarkeit begründet. Beide Parlamentsdemokratien etablierten ein besonderes Gericht, das ausschließlich für Verfassungsfragen zuständig sein sollte. ${ }^{1}$ Auch wenn sich die Kompetenzkataloge der Verfassungsgerichte in Prag und Wien recht unterschiedlich lesen, ist beiden eine wichtige Kompetenz gemeinsam:

\footnotetext{
* Schriftfassung eines Vortrages, den die Verfasserin am 19. 11. 2008 auf der vom Institut für Rechts- und Verfassungsgeschichte der Rechtswissenschaftlichen Fakultät der Universität Wien und vom Österreichischen Staatsarchiv veranstalteten Tagung "Staatsgründung und Verfassungsordnung“ in Wien gehalten hat. Die Verfasserin und die Herausgeber danken dem Institutsvorstand, Prof. Thomas Simon, für seine Zustimmung zur Veröffentlichung des Beitrages in dieser Zeitschrift.

${ }^{1}$ Aus der Fülle der Literatur zur österreichischen Verfassungsgerichtsbarkeit vgl. nur ADAMOVICH, Die Prüfung durch den Verfassungsgerichtshof; CAPPElletti, Judicial Review; Haller, Prüfung von Gesetzen; Melichar, Verfassungsgerichtsbarkeit in Österreich; ÖHLINGER, Modell der Verfassungsgerichtsbarkeit; TROPER, Kelsen. Sowie neuestens HELLER, Verfassungsgerichtshof. Zur Tschechoslowakei LANGÁŠEK, Ústavní soud; BLAHOŽ, Soudní kontrola ústavnosti; BrunNER, HOFFMANN, HOlläNDER, Verfassungsgerichtsbarkeit 13ff., 53ff.; FLANDERKA, Le contrôle de la constitutionalité; HARTMANN, Überprüfung der Gesetze; KREJČí, Principy; SCHORM, La cour constitutionnelle 11ff.; SLÁDEČEK, Ústavní soudnictví. Zusammenfassend, OSTERKAMP, Verfassungsgerichtsbarkeit.
}

die abstrakte Normenkontrolle, also die Untersuchung von Gesetzen auf ihre Verfassungsmäßigkeit losgelöst von einem bestimmten Rechtsfall. Walter Jellinek sprach rückblickend von der „österreichischen, auch in der TschechoSlowakei angenommenen Lösung". ${ }^{2}$ Angesichts der Parallelität beider Verfassungsgebungen kann man allerdings ebenso von der „österreichisch-tschechoslowakischen" Lösung einer richterlichen Verfassungskontrolle der Gesetze sprechen. ${ }^{3}$ Diese steht im Zentrum der folgenden Überlegungen.

Die Befugnis, Gesetze, die im Widerspruch zur Verfassung stehen, für nichtig bzw. ungültig zu erklären ${ }^{4}$, ist eine Garantie für den Vorrang der Verfassung und zeugt von einem ausgereiften Verfassungsbewusstsein. Sowohl die tschecho-

\footnotetext{
2 JELLINEK, Schutz des öffentlichen Rechts 38.

${ }^{3}$ Vermutungen, wonach es über Hans Kelsen und František Weyr zu einem tschechoslowakischösterreichischen Austausch über die Konstruktion des Verfassungsgerichts gekommen sei, lassen sich anhand der Archivlage nicht bestätigen. Diese These ist zuerst aufgeworfen worden von HALLER, Verfassungsgerichtshof 67 . Zur österreichischen bzw. tschechoslowakischen Priorität bei der Etablierung der Verfassungsgerichtsbarkeit siehe OSTERKAMP, Verfassungsgerichtsbarkeit $1 \mathrm{f}$.

${ }^{4}$ Für Österreich vgl. vor allem Art. 140 B-VG; für die Tschechoslowakei siehe Art. I Abs. 1 und Art. II Einführungsgesetz zur Verfassungsurkunde, Nr. 121/ 1920 Slg., und $\S \S 9$ bis 18 Verfassungsgerichtsgesetz, Nr. 162/1920 Slg.
} 
slowakische als auch die österreichische Verfassung führten eine klare Normengliederung zwischen Verfassung, Gesetzen und Verordnungen ein. Verfassungsänderungen durch bloßes einfaches Gesetz, wie sie in der Habsburgermonarchie und längere Zeit noch in Italien oder Deutschland möglich waren, kannten diese Konstitutionen nicht. ${ }^{5}$ Das Parlament war insofern in seinen demokratischen Handlungsmöglichkeiten durch verschiedene rechtstaatliche Verfahren beschränkt. Ideengeschichtlich finden sich Verfassungsvorrang und Gesetzeskontrolle bekanntlich bereits in der Spruchtätigkeit des Supreme Court der Vereinigten Staaten von Amerika seit $1803 .{ }^{6}$ Er sprach jedem einzelnen Richter die Kompetenz zu, die Übereinstimmung von Gesetzes- mit Verfassungsrecht zu überprüfen. Dieser postulierte Vorrang der Verfassung als "paramount law" war weitreichend, zeichnete sich jedoch durch eine strukturelle Begrenzung aus: Das Urteil des entscheidenden Gerichts band nur die Streitparteien und entfaltete, abgesehen von seinem Präzedenzcharakter, keine allgemeine Rechtswirkung. Das mit dem Verdikt der Verfassungswidrigkeit belegte Gesetz blieb weiter in Geltung. Auch in der Schweiz hatte sich über die Rechtsprechung des Bundesgerichts Ende des 19. Jahrhunderts eine Verfassungskontrolle herausgebildet. ${ }^{7}$ Diese Normenkontrolle bezog sich allerdings nur auf die Übereinstimmung von Kantonalrecht mit Bundesrecht, nicht auf die Verfassungsmäßigkeit von Bundesgesetzen. Der Gedanke eines durch ein Verfassungsgericht kontrollierten Bundesparlaments blieb der Schweizer Verfassungsordnung fremd, welche sich stärker auf eine Kontrolle durch Bundesrat und Bundesversammlung sowie plebiszitäre Kontrollelemente

\footnotetext{
${ }^{5}$ ADLER, Verfassung und Richteramt 115.

${ }^{6}$ Hierzu PAUlson, Constitutional Review 223ff.; STOURZH, Widerstandsrecht 15ff., 26ff.; BRUGGER, Verfassungsgerichtsbarkeit 320ff.

${ }^{7}$ GiacometTI, Schweizerisches Bundesgericht $11 \mathrm{ff}$.
}

verließ. Vor diesem Hintergrund treten die wesentlichen Merkmale der "tschechoslowakischösterreichischen Lösung" deutlich hervor: die Konzentration der Verfassungskontrolle bei einem besonderen Gericht, die Abstraktheit des Kontrollverfahrens und die allgemeine Rechtswirkung jener Entscheidung, welche die Nichtigkeit bzw. Ungültigkeit eines Gesetzes feststellt. Diese Elemente charakterisierten insgesamt das Modell der kontinentaleuropäischen „konzentrierten Verfassungsgerichtsbarkeit", wie sie nach dem Zweiten Weltkrieg vom deutschen Bundesverfassungsgericht fortentwickelt wurde. ${ }^{8}$

\section{Erster Teil: Verfassungsgerichtsbarkeit in Wien und Prag als ein Erbe der Habsburgermonarchie}

Fragt man nach historischen Entwicklungslinien für die Verfassungsgerichtsbarkeit, lassen sich zwei wichtige Motive ausmachen: erstens die politische Streitschlichtung und Friedensstiftung zwischen konkurrierenden Machtinhabern und zweitens die hierarchische Ordnung heterogener Rechtsmassen. Es überrascht daher nicht, wenn der „Prototyp“ der konzentrierten Verfassungsgerichtsbarkeit erstmals in zwei Nachfolgestaaten der Habsburgermonarchie verwirklicht wurde. Die imperiale Struktur des Habsburgerreiches hatte nicht nur zahlreiche Machtkonflikte zur Folge, sondern beruhte in administrativer Hinsicht auf verschiedenen, reich gegliederten Rechtsschichten des Reiches und seiner Teile. Am Beispiel der Föderalismusdiskussion, des Nationalitätenproblems und des Rechtstaatscharakters der Habsburgermonarchie soll zunächst

\footnotetext{
${ }^{8}$ Ein Überblick bei STARCK, WEBER, Verfassungsgerichtsbarkeit in Westeuropa 1; FALLER, Entwicklung der nationalen Verfassungsgerichte $42 \mathrm{ff}$.
} 
der Kontext skizziert werden, für dessen inhärente Spannungen und Probleme das Verfassungsgerichtsmodell eine Lösung bieten sollte.

\section{Die Föderalismusdiskussion}

Die Habsburgermonarchie war kein Bundesstaat, nach dem Willen zahlreicher Reformer sollte sie dies jedoch werden. Im verfassungsgebenden Reichstag von Kremsier traten etwa die slawischen Abgeordneten für eine nationale Landesselbständigkeit in einem föderativen Kaisertum Österreich ein. ${ }^{9}$ In den Verfassungsdebatten von 1848/1849 wurde im Rahmen solcher Föderalisierungspläne auch die Schaffung eines "Reichsgerichts" in Betracht gezogen. ${ }^{10}$ František Palacký entwarf in seinem ersten Verfassungsentwurf ein Schiedsgericht, das u.a. die Gleichheit der einzelnen Länder sichern sollte. ${ }^{11}$ Der Kremsierer Verfassungsvorschlag in seiner Endfassung, der stärker auf den Vorarbeiten von Kajetan Mayer beruhte, verankerte ein oberstes Reichsgericht, das „Streitigkeiten zwischen den Reichsländern unter einander und in Competenzstreitigkeiten der Central- und Länderregierungsgewalten"12 entscheiden sollte. Ähnliche Forderungen, die auf eine starke Reichsgerichtsbarkeit in föderalen Konflikten hinausliefen, wurden auch in der Frankfurter Paulskirche laut. Die Paulskirchenverfassung gestand dem Reichsgericht dabei äußerst differenzierte Kompetenzen zur Entscheidung föderaler Streitigkeiten $\mathrm{zu}^{13}$

\footnotetext{
${ }^{9}$ HeLFERT, Aufzeichnungen und Erinnerungen 47; RIEGER, Ústava Rakouska 602ff.

${ }^{10}$ Vgl. nur Nostitz, Entwurf einer VerfassungsUrkunde 26f.; SPRINGER, Protokolle des Verfassungsausschusses 249f.; ADAMOVICH, Kremsierer Entwurf 561ff. Siehe auch die entsprechende Passage im Entwurf von Kajetan Mayer, abgedruckt bei GOTTSMANN, Reichstag von Kremsier 133.

${ }^{11}$ RIEGER, Františka Palackého Spisy 1, 58f.

$12 \S 140$ Abs. 3 Kremsierer Entwurf.

${ }^{13}$ Hiervon umfasst waren Klagen des Bundes und der Länder wegen Verletzung der Reichsverfassung,
}

Pate für diese Verfassungsentwürfe einer schlichtenden Reichsgerichtsbarkeit im Föderalstaat stand nicht nur der amerikanische Supreme Court, der über die Schriften von Alexis de Toqueville und Robert von Mohl im deutschsprachigen Raum bekannt wurde, sondern auch die deutsche Reichstradition der Reichs- und Austrägalgerichtsbarkeit. Der richterliche Austrag schlichtete zwischen den Mitgliedstaaten des Deutschen Bundes Streitsachen, die nicht außergerichtlich beigelegt werden konnten, und war die Konsequenz für deren Verzicht auf Gewalt und kriegerische Auseinandersetzungen. ${ }^{14}$ Diese Tradition wurde zuletzt in der Wiener Schlussakte niedergelegt. ${ }^{15}$ Auf das streitentscheidende Gericht, das die Beteiligten wählen konnten, seit 1820 der oberste Gerichtshof eines Bundesstaats, sollte von Seiten der Bundesversammlung oder der Landesregierung kein politischer Einfluss ausgeübt werden. ${ }^{16}$

Ein Gericht als politischer Friedensstifter erschien auch noch den Verfassungsvätern von 1848 wünschenswert. So hieß es im Frankfurter Verfassungsausschuss, im Bundesstaat müsse der Grundsatz herrschen, dass „kein Streit zu einem Krieg führt und daß alle Streitigkeiten Entscheidung finden und nicht wie beim seligen Reichstag liegen bleiben. "17 Sowohl im Frankfurter als auch im Kremsierer Verfassungsausschuss wurden die Austrägal- und Schiedsge-

\footnotetext{
Auslegungsstreitigkeiten hinsichtlich der Reichsverfassung zwischen den beiden Kammern des Bundesparlaments und der Bundesregierung sowie politische und privatrechtliche Streitigkeiten der Länder untereinander sowie der Grundrechtsschutz. Vgl. §126 a bis d Paulskirchenverfassung. Hierzu KüHNE, Reichsverfassung der Paulskirche 199ff., 384ff.; SCHEUNER, Überlieferung der deutschen Staatsgerichtsbarkeit $28 \mathrm{ff}$.

14 WELCKER, Schiedsgerichte 778ff.

${ }^{15}$ MERZBACHER, Austrägalinstanz 273f.

${ }^{16}$ Siehe Art. XXII Wiener Schlussakte vom 15. 5. 1820.

${ }^{17}$ DROYSEN, Verhandlungen des Verfassungsausschusses 1, 124; zu Kremsier vgl. JelLINEK, Verfassungsgerichtshof für Österreich 62.
} 
richtsbarkeit als eine Kompetenz des Reichsgerichts verhandelt, schließlich überließ man dem Reichsgericht jedoch nur einen kleinen Spielraum als Schiedsrichter in politischen Streitigkeiten. ${ }^{18}$ Stattdessen setzte man vor allem im Bereich der konkurrierenden Reichs- und Landesgesetzgebung auf die Verrechtlichung des politischen Konflikts und die einheitliche Rechtsprechung durch ein oberstes Gericht. Mit einem starken Reichsgericht erhoffte man sich auch eine Stärkung der Zentralgewalt. ${ }^{19}$ Das institutionelle Vorbild für diese Lösung, der Supreme Court, hatte bei den Machtstreitigkeiten zwischen Bundesgebilde und Gliedstaaten ebenfalls zugunsten der Zentralgewalt Stellung genommen und den Verfassungsvorrang aufgrund des "judicial review" lange Zeit ausschließlich als Schranke für die Ländergewalt angewandt.

Die Idee einer föderalen Verfassungsgerichtsbarkeit blieb in den juristischen Debatten in Österreich auch nach dem Scheitern der Verfassungsgebung in Kremsier lebendig. ${ }^{20}$ Hierbei gewann das amerikanische Vorbild und die Idee eines zentralen Höchstgerichts zunehmend an Gewicht. ${ }^{21}$ Der Charakter eines vor allem politisch geprägten Schiedsgerichts und „eines höchsten Moderators zwischen der Reichsgewalt den Ländern“ wurde von zeitgenössischen Kommentatoren demgegenüber als „utopisch“ abgetan. ${ }^{22}$

\section{Das Nationalitätenproblem}

Anders als in den deutschen Ländern oder in den Vereinigten Staaten kreisten die Debatten um eine Föderalisierung der Habsburgermonar-

\footnotetext{
${ }^{18}$ FALLER, Verfassungsgerichtsbarkeit in Frankfurter Reichverfassung 842.

${ }^{19}$ LEMAYER, Rechtsschutz im öffentlichen Rechte 18.

${ }^{20}$ Siehe hierzu vor allem die Schriften von. EÖTVÖs, Herrschende Ideen 2, 381ff.; JELLINEK, Verfassungsgerichtshof für Österreich; RENNER, Kampf um den Staat 241.

${ }^{21}$ JELLINEK, Verfassungsgerichtshof für Österreich 56.

${ }^{22}$ LEMAYER, Rechtsschutz im öffentlichen Rechte 18.
}

chie nicht ausschließlich um das Verhältnis zwischen den Gliedstaaten und dem übergeordneten Gemeinwesen. Hinter den Reformierungsbestrebungen im österreichischen Kaisertum stand das drängende Nationalitätenproblem. Die Schriften des Sozialdemokraten und späteren österreichischen Bundespräsidenten Karl Renner machen deutlich, dass sich in der Habsburgermonarchie der Jahrhundertwende die primär politische, schiedsrichterliche Funktion der Verfassungsgerichtsbarkeit keineswegs erledigt hatte. Angesichts einer zersplitterten Bevölkerungslandschaft, die vielerorts national durchmischte Gebiete zeigte, blieb für ihn fraglich, ob eine föderale territoriale Neuordnung die um politischen Einfluss konkurrierenden Nationalitäten tatsächlich befrieden könne. ${ }^{23}$ Die theoretische Verbindung von Nationalitätenfrage und Verfassungsgerichtsbarkeit war daher für ihn von besonderer Anziehungskraft.

Ein interessantes Modell für einen solchen Nationalitätengerichtshof findet sich wiederum bereits bei Palacký. Im Kremsierer Verfassungsausschuss schlug er vor, neben der Gleichheit der Länder auch die Gleichheit der Nationen zu garantieren und strittige Fragen zwischen den Provinzen und Nationalitäten durch ein Gericht zu lösen. ${ }^{24}$ Dieses Gericht solle von Fall zu Fall und nur dann tätig werden, wenn der Reichstag den Konflikt nicht selbst entscheide. Palackýs Vorschlag stand ganz im Zeichen der politischen Schiedsgerichtsbarkeit. Im Kremsierer Verfassungsentwurf wurde dieser Vorschlag nur in abgeschwächter Form übernommen: Für die Landesverfassungen der „Reichsländer gemischter Nationalität" wurde die Möglichkeit eröffnet, ein „Schiedsgericht" für Angelegenheiten „von

\footnotetext{
${ }^{23}$ RENNER, Kampf um den Staat 32-35; DERS, Selbstbestimmungsrecht 72-74.

${ }^{24} \S \S 56$ bis 59 des ersten Verfassungsentwurfs von Palacký, vgl. RIEGER, Františka Palackého Spisy 1, $57 \mathrm{ff}$.
} 
rein nationeller Art" einzurichten. ${ }^{25}$ Nach dem Ende des Konstitutionalismus in Kremsier wurden keine vergleichbaren Institutionen etabliert, ein Klagerecht der Nationalitäten scheiterte an der fehlenden Anerkennung ihrer Rechtspersönlichkeit. ${ }^{26}$

Die Schrift Karl Renners „Der Kampf der österreichischen Nationen um den Staat" aus dem Jahr 1902 führte Verfassungsgerichtsbarkeit, Föderalismus und Nationalitätenproblem schließlich dezidiert zusammen. Nach Renners Auffassung sollte der politische Machtkampf der Nationalitäten durch die Neutralität einer richterlichen Instanz entschärft werden: „Das Reichsgericht interpretiert die Verfassungsgesetze, und alle Organe sind an diese Interpretation gebunden. Es entscheidet, auch wenn keine verfassungsrechtlichen Fragen vorliegen, in allen Rechtstreitigkeiten des öffentlichen Rechts zwischen Nation und Nation, sowie zwischen Staat und Nation." 27 Diese bewusste Ausdehnung der Rechtsprechungskompetenz auf „alle Rechtsstreitigkeiten des öffentlichen Rechts" auch ohne Verfassungsbezug ist von einer klaren Intention getragen, der Befriedung des nationalpolitischen Kampfes. Renners Lösungsvorschlag setzt dabei auf eine Verrechtlichung der nationalen Streitigkeiten: die juristische Anerkennung der Nation einerseits und die rechtliche Konfliktbereinigung andererseits. ${ }^{28}$ Über eine bloß territoriale Neugliederung des Staates weit hinausgehend, verband Renner sein Föderalismusmodell mit bedeutenden Kollektivrechten für nationale Gruppen und fand hierfür den sprechenden Namen „Nationalitätenbundesstaat". ${ }^{29}$ Darin erfüllte das Verfassungsgericht nicht nur eine rechtlich-koordinierende Aufgabe im hierarchisch-föderalen Staatsaufbau, sondern

\footnotetext{
${ }^{25} \S 113$ Kremsierer Entwurf.

${ }^{26}$ MALFÉR, Österreichische Verfassungsdiskussion 72.

${ }^{27}$ RenNER, Kampf um den Staat 241.

${ }^{28}$ RENNER, Selbstbestimmungsrecht 24ff., $292 \mathrm{ff}$.

${ }^{29}$ Ebd., 294.
}

war zugleich Schiedsinstanz zwischen den konkurrierenden Volksgruppen.

Unmittelbar nach dem Zerfall der Habsburgermonarchie erlebte die Idee eines Nationalitätengerichtshofs in der Tschechoslowakei eine kurze Renaissance. Nach der Anerkennung der Tschechoslowakei durch die Großmächte legte die deutschböhmische Friedensdelegation in St. Germain, die lange einen Anschluss der deutschen Randgebiete an Österreich bzw. Deutschland favorisiert hatte, im Juni 1919 erstmals einen eigenen Verfassungsentwurf vor. Darin empfahl sie für den neuen tschechoslowakischen Staat eine kantonale Struktur nach Schweizer Vorbild. Während die nationalen Kantone großzügige Selbstverwaltungsrechte genießen und dem Gesamtstaat nur subsidiäre Regelungskompetenzen zukommen sollten, war als Bewahrer dieser Machtverteilung ein „Gerichtshof für verfassungsrechtliche Streitigkeiten" bestellt, der sich nach dem nationalen Proporzprinzip zusammensetzen sollte. ${ }^{30}$ Da man auf tschechoslowakischer Seite Sezessionsbestrebungen fürchtete, obsiegte jedoch ein zentralistischer Staatsaufbau. Die deutschen Forderungen nach einem Nationalitätengerichtshof setzten sich nicht durch.

\section{Die Rechtstaatsidee}

Fragt man nach weiteren Ursachen, die gerade in Wien und Prag die Verfassungsgerichtsbarkeit begünstigten, sollte man einen dritten Punkt nicht außer Acht lassen: die konstitutionelle Rechtstaatsidee. Nach der gewaltsamen Auflösung der verfassungsgebenden Versammlung in Kremsier und dem Scheitern der Paulskirchenverfassung war der Traum der liberalen und demokratischen Reformer von einem umfassenden Verfassungsdokument vorerst zu Ende. In

\footnotetext{
${ }^{30}$ Bericht über die Tätigkeit der deutschösterreichischen Friedensdelegation in St. Germain-en-Laye I, 338ff.
} 
der zweiten Hälfte des 19. Jahrhunderts entwickelte sich das Verfassungsrecht in Österreich im Unterschied zur Bismarckschen Verfassung für das Deutsche Reich - jedoch über ein bloßes staatliches Organisationsrecht weit hinaus.

Die Basis für dieses zwar fragmentarische, doch wichtige materielle Regelungen enthaltende Verfassungsrecht bildeten die Staatsgrundgesetze von 1867. Das Staatsgrundgesetz über die politischen Rechte der Staatsbürger garantierte nicht nur ein Mindestmaß politischer Freiheitsrechte, sondern schuf auch Regelungen für die religiöse und nationale Gleichberechtigung. ${ }^{31}$ Die Dezemberverfassung ließ es nicht bei einer programmatischen Verkündung dieser Grundrechte bewenden, sondern installierte zu ihrem Schutz öffentliche Gerichte: das Reichsgericht und einen zentralen Verwaltungsgerichtshof. ${ }^{32}$ Während das Verwaltungsgericht grundrechtsverletzende Akte der Verwaltungsbehörden aufheben konnte, hatten die Entscheidungen des Reichsgerichts, insbesondere zum nationalen Gleichbehandlungsgebot, keine kassatorische Wirkung. ${ }^{33}$ Die informelle Wirkung der Reichsgerichtsurteile in nationalitätenpolitischen Fragen darf dennoch nicht unterschätzt werden: Das Reichsgericht wandte die aus Art. 19 des Staatsgrundgesetzes über politische Rechte abgeleiteten Nationalitätenrechte unmittelbar an und gestand die Befugnis zur Verfassungsbeschwerde großzügig Gemeinden, Vereinen und anderen Trägern der autonomen Verwaltung zu. ${ }^{34}$ Eine solche Nationalitätenrechtsprechung

\footnotetext{
${ }^{31}$ STOURZH, Georg Jellinek 249.

${ }^{32}$ Staatsgrundgesetz vom 21. 12. 1867 über die Einsetzung eines Reichsgerichtes, RGBl. 143/1867 sowie Art. 15 des Staatsgrundgesetzes vom 21. 12. 1867 über die richterliche Gewalt, RGBl. 144/1867. Die Verwaltungsgerichtsbarkeit nahm allerdings erst 1876 ihre Tätigkeit auf. STOURZH, Georg Jellinek 250.

${ }^{33}$ STOURZH, Georg Jellinek $249 f$.

${ }^{34}$ Hierzu STOURZH, Gleichberechtigung der Nationalitäten $53 f f$.
}

war im europäischen Kontext ohne Beispiel. ${ }^{35}$ Aufgrund dieser umfangreichen, wegen der gesetzlichen Regelung jedoch lückenhaften Rechtsprechung, verstummten in Österreich jene Stimmen nie, die einen erweiterten Verfassungsschutz für die politischen Rechte forderten. ${ }^{36}$

Auch das Problem eines Vorrangs des Verfassungsrechts geriet immer wieder in den wissenschaftlichen Fokus. Die Dezemberverfassung von 1867 hatte eine wesentliche Frage offengelassen: das Verhältnis von Reichs- und Landesrecht. ${ }^{37}$ Die Kompetenzen der Zentralgewalt wurden in Umkehrung der bisherigen Ordnung taxativ aufgelistet, im Zweifel waren die Landtage zuständig. Eine Kollisionsregel fehlte, nachdem das Abgeordnetenhaus die Schlichtung der Zuständigkeitsstreitigkeiten durch den Kaiser abgelehnt hatte. ${ }^{38}$ In der Praxis kam es in der Folge zu zahlreichen gegenseitigen Kompetenzüberschreitungen, wobei insbesondere das Reich zur Ausweitung seiner Zuständigkeiten neigte. ${ }^{39}$ Bei Geltungskonflikten kollidierender Normen fand die Regel lex posterior derogat priori Anwendung, doch empfand man dieses Vorgehen als unbefriedigend. ${ }^{40}$

Jellineks richtungweisende Schrift „Ein Verfassungsgerichtshof für Österreich“ konzentrierte daher die föderalen Kompetenzkonflikte beim Reichsgericht, das ohnehin über eine rudimentäre Kompetenzgerichtsbarkeit verfügte. ${ }^{41}$ Das Reichsgericht sollte zu einer juristischen Ent-

\footnotetext{
${ }^{35}$ STOURZH, Georg Jellinek 257.

${ }^{36}$ HugelmanN, Das österreichische Reichsgericht $458 \mathrm{ff}$.

${ }^{37}$ Hierzu WiEDERIN, Bundesrecht und Landesrecht $63 \mathrm{ff}$.

${ }^{38}$ Ebd., $61 \mathrm{ff}$.

${ }^{39}$ Ebd., 64.

${ }^{40}$ Haller, Prüfung von Gesetzen 31.

${ }^{41}$ JELLINEK, Verfassungsgerichtshof für Österreich 4851. Dazu STOURZH, Georg Jellinek 254ff; Noll, Forderung nach Verfassungsgerichtshof 261ff.; WYDUCKEL, Entwicklung der Verfassungsgerichtsbarkeit 277ff., 281ff. sowie KeRSTEN, Mehrheit und Minderheit 221ff.
} 
scheidungsinstanz von Konflikten zwischen Reichs- und Landesrecht werden. ${ }^{42}$ Dabei sollte der Vorrang der Verfassung gesichert und kontrolliert werden. ${ }^{43}$ In der Schrift von Jellinek wurde die Auffassung vom Reichsgericht als einer friedensstiftenden, letztlich politisch agierenden Instanz im föderalen Konflikt, welche noch die Debatten in Frankfurt und Kremsier beherrscht hatte, endgültig abgelöst von der Idee eines rechtstaatlichen Garanten der Rechtseinheit. ${ }^{44}$ Es ging nicht mehr um die Gefahr kriegerischer Machtkonflikte zwischen einzelnen Gliedstaaten, sondern um die verfassungsmäßige Organisation konfligierender Rechtsmassen. Mit ähnlicher Stoßrichtung argumentierte im Anschluss an Jellinek Heinrich Jaques ${ }^{45}$ sowie später František Weyr und Adolf Merkl ${ }^{46}$. Diese Überlegungen fanden Eingang in das österreichische Bundesverfassungsgesetz von 1920. In der Tschechoslowakei, welche als Einheitsstaat organisiert war, übernahm man die Kompetenzgerichtsbarkeit nicht. Der Jellineks Überlegungen zugrundeliegende Gedanke einer rechtstaatlichen Verfassungsbindung von Gesetzgebung und Verwaltung wurde jedoch zentral auch für die tschechoslowakische Verfassung und Verfassungsgerichtsbarkeit. ${ }^{47}$

\footnotetext{
42 JELLINEK, Verfassungsgerichtshof für Österreich $27 \mathrm{ff}$.

${ }^{43}$ Dazu Haller, Prüfung von Gesetzen 26ff.

${ }^{44}$ Die Idee der Rechtseinheit leitete sich bei Jellinek aus der Unteilbarkeit des kaiserlichen Willens ab, hierzu WIEDERIN, Bundesrecht und Landesrecht 65 .

${ }^{45}$ Allerdings gegen den präventiven Ansatz der Gesetzeskontrolle Jellineks gewandt; HALLER, Prüfung von Gesetzen 28f.

${ }^{46}$ HALler, Prüfung von Gesetzen 30ff.

${ }^{47}$ Bereits die Gesetzesbegründung zum Verfassungsgerichtsgesetz nimmt auf Jellinek Bezug; Drs. 2426 der Tschechoslowakischen Nationalversammlung. Vgl. OsTERKAMP, Verfassungsgerichtsbarkeit 11ff., 116ff., 124ff.
}

\section{Zweiter Teil: Das tschechoslowakische Modell der Verfassungsgerichtsbarkeit}

Von den drei genannten Faktoren, welche die ideengeschichtliche Entwicklung einer konzentrierten Verfassungsgerichtsbarkeit in der Habsburgermonarchie befördert hatten, blieb in der tschechoslowakischen Verfassung vom 29. Februar 1920 nur ein Moment sichtbar: die Rechtstaatsidee. Die Verfassungsurkunde etablierte einen Geltungsvorrang der Verfassung vor den Gesetzen sowie eine Gesetzesbindung der Verwaltung. Die Zuständigkeit des tschechoslowakischen Verfassungsgerichts beschränkte sich auf einen einzigen gewichtigen Kern: Die Verfassungskontrolle höherrangiger Rechtsnormen. Von den Fragen eines föderalen Staatsaufbaus oder der Garantie nationalitätenpolitischer Gleichberechtigung war sie demgegenüber gänzlich losgelöst. Beim Verfassungsgericht waren zwei Kontrollverfahren angesiedelt: zum einen untersuchte es in einem Antragsverfahren Gesetze auf ihre Verfassungsmäßigkeit und erklärte diese gegebenenfalls für nichtig; zum anderen überprüfte es von Amts wegen die sogenannten vorläufigen Verfügungen des Ständigen Ausschusses, die den Charakter von Notverordnungen hatten. Anders als der Wiener Verfassungsgerichtshof war das Verfassungsgericht nicht zur Überprüfung von Rechtsverordnungen berechtigt, hierzu war die ordentliche Gerichtsbarkeit berufen.

Der Kompetenzumfang des Prager Verfassungsgerichts war im Vergleich zu seinem Wiener Pendant daher weitaus geringer. Dies lag nicht nur am Fehlen einer Föderalstruktur in der Tschechoslowakei, weshalb zahlreiche verfassungsrechtliche Zuständigkeiten im tschechoslowakischen Kontext hinfällig waren, sondern auch daran, dass gewichtige gewaltenteilige Kontrollmechanismen auf andere Verfassungsorgane verteilt waren. So gab es keine Verfassungsbeschwerde vor dem Verfassungsgericht, 
der Schutz der politischen Grundrechte gegen rechtswidrige Eingriffe der Verwaltung war beim Obersten Verwaltungsgericht angesiedelt. ${ }^{48}$ Die Wahlgerichtsbarkeit, die über die Rechtmäßigkeit von Wahlergebnissen und verfahren sowie den Verlust des parlamentarischen Mandats entschied und in Österreich vom Verfassungsgericht ausgeübt wurde, war einem besonderen Wahlgericht übertragen. ${ }^{49}$ Die Rechtsverordnungen wurden im Wege des allgemeinen richterlichen Prüfungsrechts kontrolliert, hier erlangte insbesondere die Rechtsprechung des Obersten Verwaltungsgerichts Bedeutung. ${ }^{50}$ Die Gesetzgebung unterlag nicht nur der Verfassungskontrolle durch das Verfassungsgericht, sondern auch dem innerparlamentarischen Kontrollmechanismus des Zweikammersystems und dem aufschiebenden Vetorecht des Präsidenten der Republik. ${ }^{51}$ Auf die Ministeranklage, wie sie das österreichische BundesVerfassungsgesetz noch vorsah ${ }^{52}$, hatte man in der Tschechoslowakei zugunsten eines parlamentarischen Misstrauensvotums gegenüber der Regierung verzichtet ${ }^{53}$.

\section{Die Verfassungskontrolle der Gesetze}

Das Verfassungsgericht wurde als ein rechtstaatliches Gegengewicht zum Parlament errichtet. Das Ziel, die Übereinstimmung der Gesetze mit der Verfassung zu garantieren, hob der erste Verfassungsgerichtspräsident Karel Baxa, der gleichzeitig Oberbürgermeister der Stadt Prag war, in seiner Eröffnungsrede des Verfassungsgerichts am 7. November 1922 hervor: „Unsere demokratische Verfassung hat diese Institution [das Verfassungsgericht] im Interesse der Sicherung der Rechtsordnung und damit auch im

\footnotetext{
${ }^{48}$ Ebd., 188ff.

${ }^{49}$ Ebd., 17ff.

${ }^{50}$ Ebd., 60ff.

${ }^{51}$ Ebd., 58ff.

${ }^{52}$ Art. 142 B-VG.

53 ADLER, Parlamentarisches Regierungssystem 299.
}

Interesse der Bürger eingeführt, um zu gewährleisten, dass die Grundgesetze des Staates nicht ohne die in der Verfassung vorgeschriebenen Kautelen geändert werden. ${ }^{\text {" } 54}$ Baxas Worte machen deutlich, dass das primäre Anliegen der tschechoslowakischen Verfassungsgerichtsbarkeit die objektive Sicherung der Verfassungsordnung war. Erst an zweiter Stelle standen die subjektiven Interessen der Staatsbürger. Diese Einschätzung gilt im Übrigen auch für die Verfassungskontrolle der Gesetze in Österreich ${ }^{55}$, nicht aber für die österreichische Verfassungsgerichtsbarkeit insgesamt. Im Bereich der Verfassungsbeschwerde spielte das Wiener Gericht eine Vorreiterrolle und verhalf dem subjektiven Grundrechtsschutz zu einem Durchbruch.

Als weiteres Charakteristikum der tschechoslowakischen Verfassungskontrolle kann neben der Ausrichtung auf die objektive Rechtsordnung ihre Abstraktheit und Allgemeinheit gelten. Versehen mit der Rechtstellung eines „negativen Gesetzgebers" konnte das Verfassungsgericht die Gesetze grundsätzlich in jeder sachlichen Hinsicht auf ihre Verfassungsmäßigkeit überprüfen. Anders als das amerikanische Modell einer inzidenten Verfassungskontrolle im laufenden Verfahren der ordentlichen Gerichtsbarkeit war die Gesetzeskontrolle im tschechoslowakischen Modell - stärker noch als in Österreich - von den konkreten Rechtsfällen losgelöst. Damit das Verfassungsgericht tätig werden konnte, bedurfte es allerdings eines begründeten schriftlichen Antrags. ${ }^{56}$

\footnotetext{
${ }^{54}$ Manuskript der Eröffnungsrede, in: NA, ÚS, Kart. 6, Heft 2.

${ }^{55}$ MARCIC, Verfassung und Verfassungsgericht 155; CAPPELLETTI, Judicial Review 71ff.

${ }^{56}$ Weiteres Zulässigkeitserfordernis der Verfassungskontrolle war eine dreijährige Präklusionsfrist. Drei Jahre nach Verkündung des Gesetzes wurde es auch vor dem Verfassungsgericht unanfechtbar. Dieses Erfordernis wird im Rahmen dieser Studie außer acht gelassen. Vgl. dazu OsTERKAMP, Verfassungsgerichtsbarkeit 32ff.
} 


\section{Das Antragsverfahren}

Das Antragsverfahren ist der entscheidende Mechanismus für das Gelingen der abstrakten Verfassungskontrolle und das Gelingen der konzentrierten Verfassungsgerichtsbarkeit insgesamt. Dass für die Verfassungskontrolle der Gesetze ein Antragsverfahren vorgesehen ist, dient der Machtbegrenzung des Verfassungsgerichts. Es soll nicht von sich aus tätig und die Gesetze des demokratisch legitimierten Parlaments in Zweifel ziehen können: Der Garant der Rechtstaatlichkeit soll sich nicht ohne weiteres über den Repräsentanten der Demokratie erheben können. Anders als in Österreich konnte das tschechoslowakische Verfassungsgericht selbst dann nicht über die Verfassungsmäßigkeit eines Gesetzes entscheiden, wenn es die Entscheidungsvoraussetzung in einem anderen Fall war. ${ }^{57}$

Es ist lohnend, das tschechoslowakische Antragsverfahren mit dem österreichischen zu vergleichen. Der Kreis der Antragsberechtigten, die beim Verfassungsgericht ein abstraktes Gesetzeskontrollverfahren anstrengen konnten, war hier im Vergleich zur österreichischen Regelung weiter gezogen. Konnten zunächst vor dem Wiener Verfassungsgerichtshof Landesgesetze nur auf Antrag der Bundesregierung bzw. Bundesgesetze auf Antrag einer oder mehrerer Landesregierungen geprüft werden, so waren vor dem Prager Verfassungsgericht insgesamt sechs Verfassungsorgane antragsbefugt. Es handelte sich um die beiden Kammern des Parlaments, das Wahlgericht, den Landtag der Karpathenukraine sowie das Oberste Gericht und das Oberste Verwaltungsgericht. Von diesen sechs Akteuren trat der karpathenukrainische Landtag im Widerspruch zu verfassungs- und völker-

\footnotetext{
${ }^{57}$ Vgl. zur Gerichtspraxis OSTERKAMP, Verfassungsgerichtsbarkeit 12, 183ff. Zu dieser Kompetenz als einem Motor der Verfassungsgerichtsbarkeit vgl. ÖHLINGER, Modell der Verfassungsgerichtsbarkeit 591.
}

rechtlichen Garantien allerdings niemals ins Leben.

Mit Blick auf den Kreis möglicher Antragsteller zeigt sich bei der tschechoslowakischen Lösung gegenüber der österreichischen Regelung ein wichtiger konstruktiver Vorteil: Während im österreichischen Gesetzeskontrollverfahren Anträge nur von politischen Verfassungsorganen zulässig waren, ließ das tschechoslowakische Verfahren auch solche von Justizorganen zu. Mit den beiden obersten Gerichten waren dadurch Verfassungsorgane in das Gesetzeskontrollverfahren einbezogen, die bei ihrer täglichen Arbeit mit Fragen der Verfassungsmäßigkeit bestimmter gesetzlicher Regelungen konfrontiert waren. Im Jahre 1929 wurde die österreichische Regelung dahingehend ergänzt, dass nunmehr ebenfalls der Oberste Gerichtshof und der Verwaltungsgerichtshof antragsbefugt waren. ${ }^{58}$ Sie folgte insoweit dem tschechoslowakischen Modell. 59

Für das Funktionieren oder Nicht-Funktionieren der Verfassungsgerichtsbarkeit ist allerdings nicht nur die rechtliche Konstruktion des Antragsverfahrens, sondern auch das politische Umfeld verantwortlich. Im Bereich der Verfassungskontrolle der Gesetze waren dem tschechoslowakischen Verfassungsgericht lange Zeit die Hände gebunden. Keines der berechtigten Verfassungsorgane stellte bei Zweifeln an der Verfassungsmäßigkeit eines Gesetzes den erforderlichen Antrag. Erst im Jahr 1936 stießen die obersten Gerichte ein Gesetzeskontrollverfahren an. Dabei zeigt ein Blick in die zeitgenössische rechtswissenschaftliche Literatur und die Tagespresse, dass gerade in politisch sensiblen Bereichen wie dem Sprachenrecht oder bei staatlichen Wirtschaftslenkungsmaßnahmen die Ver-

\footnotetext{
${ }^{58}$ Vgl. § 60 Zweite B-VG Novelle, BGB1 392/1929.

${ }^{59}$ Ein bewusster Verfassungstransfer wurde meines Wissens lediglich auf tschechoslowakischer Seite vermutet. Vgl. ADLER, Verfassung und Richteramt 116.
} 
fassungsmäßigkeit des gesetzgeberischen Handelns mitunter heftig umstritten war. Der Widerspruch zwischen der theoretischen Möglichkeit des Verfassungsgerichts, ein Gesetz in jeder Hinsicht auf seine Verfassungsmäßigkeit hin zu untersuchen, und seiner praktischen Unfähigkeit, von dieser Befugnis Gebrauch zu machen, war den Zeitgenossen bewusst. Sie drangen auf eine stärkere Anlehnung an das österreichische Muster bzw. führten das Daniederliegen der Gesetzeskontrolle darauf zurück, dass der Kreis der Antragsberechtigten zu eng sei. ${ }^{60}$

\section{Schattenseiten der Abstraktheit}

Den möglichen Antragstellern fehlte die Motivation, vor dem Verfassungsgericht ein Kontrollverfahren anzuregen. Die föderalen und nationalitätenpolitischen Komponenten, die ideengeschichtlich für die konzentrierte Verfassungsgerichtsbarkeit so fruchtbar gewesen waren, fehlten im tschechoslowakischen Gesetzeskontrollverfahren. Hier brachte auch das Zweikammersystem keine Abhilfe. Anders als im Verfassungsausschuss der tschechoslowakischen Revolutionären Nationalversammlung ursprünglich einmal vorgesehen, repräsentierten die beiden Kammern, das Abgeordnetenhaus und der Senat, keine unterschiedlichen oder gar widerstreitenden nationalen, sozialen, territorialen oder wirtschaftlichen Interessen. ${ }^{6}{ }^{1}$ Da sich die zwei parlamentarischen Kammern organisatorisch nur geringfügig voneinander unterschieden und die Wahlen zu den Kammern gleichzeitig stattfanden, entsprach sich die Sitzverteilung der politischen Parteien geradezu

\footnotetext{
${ }^{60}$ So wurde u.a. eine Konzentration der Verfassungsbeschwerde beim Verfassungsgericht erwogen; OsTERKAMP, Verfassungsgerichtsbarkeit 26ff.

${ }^{61}$ Gegen das Zweikammersystem hatte sich vorrangig die Sozialdemokratie gewandt, auf deren Einwände das schließlich umgesetzte Modell zurückgeht; KYSELA, Dvoukomorové systémy 361ff.; BROKLOVÁ, První československá ústava 11f.
}

spiegelbildlich: Die Gesetze erließ zwar das Abgeordnetenhaus, doch wurden sie politisch vom Senat mitgetragen. $\mathrm{Zu}$ produktiven Differenzen zwischen den Kammern, welche zu einem Motor für die Klärung von Verfassungsfragen hätten werden können, kam es nicht. ${ }^{62} \mathrm{Ihr}$ Antragsrecht vor dem Verfassungsgericht lief ins Leere. Mit dem Parlament politisch eng verbunden war auch das Wahlgericht. Zwar durften die Wahlrichter nicht gleichzeitig Abgeordnete sein, dennoch gelang es den politischen Mehrheitsparteien, auf die Rechtsprechung des Wahlgerichts Einfluss zu nehmen. Dessen Tätigkeit wurde von einem führenden Rechtswissenschaftler als eines der "traurigsten“ Kapitel des tschechoslowakischen Rechtslebens bezeichnet ${ }^{63}$; an einen Antrag auf Gesetzeskontrolle vor dem Verfassungsgericht war nicht zu denken.

Aufgrund dieser politischen Verflechtungen ist es wenig überraschend, dass es ausschließlich die beiden obersten Gerichte waren, die beim Verfassungsgericht einen Antrag zur Überprüfung eines Gesetzes einbrachten. Im Jahr 1936 legten sie dem Verfassungsgericht zwei Gesetze zur Überprüfung vor, die wichtige legislative Regelungsbefugnisse im Wege einer Blankettermächtigung vom Parlament auf die Regierung übertrugen. ${ }^{64}$ Der spätere Teilerfolg vor Gericht kann jedoch nicht darüber hinwegtäuschen, dass die obersten Gerichte auch schon vor 1936 reichlich Gelegenheit hatten, das Verfassungsgericht anzurufen.

Ihre Untätigkeit hatte mehrere Ursachen. Unmittelbar nach Republikgründung spielte sicherlich eine Rolle, dass die obersten Gerichte die Tätig-

\footnotetext{
${ }^{62}$ Ein Antragsrecht einer parlamentarischen Minderheit vor dem Verfassungsgericht, wie es sich etwa in älteren Vorschlägen von Jellinek oder Kelsen fand, war nicht vorgesehen; OSTERKAMP, Verfassungsgerichtsbarkeit $15 \mathrm{f}$.

${ }^{63}$ Hoetzel, Ústava 479.

${ }^{64}$ Zur Geschichte dieser Anträge vor dem Verfassungsgericht und dessen Entscheidung, vgl. OsTERKAMP, Verfassungsgerichtsbarkeit 154ff, 161ff.
} 
keit des Parlaments, das als Symbol und Repräsentant des neu gegründeten tschechoslowakischen Staates besondere Anerkennung genoss, nicht delegitimieren wollten. Unter den Verfassungsrichtern sprach man aber auch von der „Eitelkeit“ der obersten Richter „ihre Rechtsanschauung einer Überprüfung des Verfassungsgerichtes nicht aussetzen zu wollen" 65 bzw. ihrer Furcht, durch eine gegenteilige Ansicht des Verfassungsgerichtes "desavouiert" ${ }^{\prime \prime 6} \mathrm{zu}$ werden. Eine neuartige Institution wie das Verfassungsgericht musste sich gegenüber der ordentlichen Gerichtsbarkeit erst behaupten.

Die Zurückhaltung der obersten Richter hatte aber auch strukturelle Gründe: die Abstraktheit der Gesetzeskontrolle mit ihrer auf die Spitze getriebenen Loslösung von konkreten Rechtsfällen. Auch das verdeutlicht der Verfassungsvergleich mit Österreich. Die tschechoslowakische Regelung gestattete es den obersten Richtern, auch unabhängig von konkreten Rechtssachen Zweifelsfälle über die Verfassungsmäßigkeit eines Gesetzes vor das Verfassungsgericht zu bringen. Sie ging insofern über das 1929 eingeführte Antragsrecht der obersten Gerichte in Österreich hinaus, das abhängig davon war, dass das angegriffene Gesetz die „Voraussetzung eines Erkenntnisses des antragstellenden Gerichtshofes ${ }^{\prime 67}$ bildete. Die Entscheidung des tschechoslowakischen Verfassungsgerichts entfaltete hingegen keine Wirkung in der konkreten, bei den obersten Gerichten anhängigen Sache. Der betroffene Senat, der bei seiner Entscheidungsfindung an der Verfassungsmäßigkeit eines zugrunde zu legenden Gesetzes Zweifel hegte oder hierauf von den Streitparteien hingewiesen wurde, stellte nicht selbst den Antrag beim Verfassungsgericht, vielmehr ent-

\footnotetext{
${ }^{65}$ KREJČí, Prüfung der Verfassungsmäßigkeit 43.

${ }^{66}$ Protokoll der nichtöffentlichen Sitzung des Verfassungsgerichts vom 19.10.1923, in: NA, ÚS, Kart. 1 Heft Pres I, Az Úst 102/23-pres 1/15.

${ }^{67}$ Art. 140 Abs. 1 B-VG.
}

schied hierüber die Plenarsitzung des obersten Gerichts. ${ }^{68}$ Bis zu deren Entscheidung durfte der Senat die anhängige Sache nicht ruhen lassen und erst recht nicht das Urteil des Verfassungsgerichts abwarten. Dahinter stand die Überlegung des Verfassungsgebers, dass den Streitparteien vor den obersten Gerichten kein Mittel an die Hand gegeben werden sollte, „über die Einwendung der Verfassungswidrigkeit eines Gesetzes auch die Unterbrechung des Verfahrens zu fordern" ${ }^{\prime \prime}{ }^{69}$ Diese Konstruktion entsprach der Systematik der tschechoslowakischen Verfassungsgerichtsbarkeit, die zuallererst auf die objektive Sicherung der Rechtsordnung setzte und in der ein Schutz individueller Interessen lediglich beiläufiges Ergebnis der Gesetzeskontrolle war. Für die objektive Sicherung der Rechtsordnung stand im Vordergrund, dass ein verfassungswidriges Gesetz überhaupt als solches für nichtig bzw. ungültig erklärt wurde, nicht aber, dass diese Entscheidung auf den konkreten Rechtsfall zurückwirkte, der den Anlass zur Gesetzeskontrolle gegeben hatte. ${ }^{70}$

Diese strenge Abstraktheit der Gesetzeskontrolle hatte letztlich zur Folge, dass den obersten Richtern in der Tschechoslowakei jeglicher Anreiz zur Verfassungskontrolle fehlte. Das erwähnte Gesetzeskontrollverfahren setzten sie erst in einem Moment in Gang, als ihre eigenen Berufsinteressen betroffen waren. ${ }^{71}$ Die österreichische Lösung erwies sich demgegenüber als pragmatischer. Durch die Verfassungsnovelle von 1929

\footnotetext{
${ }^{68} \S 10$ des Verfassungsgerichtsgesetzes, Nr. 162/1920 Slg.

${ }^{69}$ Motivenbericht zur tschechoslowakischen Verfassungsurkunde, Drs 2421/1920 der Tschechoslowakischen Nationalversammlung.

${ }^{70}$ Auf die abweichenden akademischen Ansichten, welche den Urteilen des Verfassungsgerichts - abweichend vom Motivenbericht des Gesetzgebers - eine Wirkung ex tunc zusprachen, kann an dieser Stelle nicht eingegangen werden; dazu OsTERKAMP, Verfassungsgerichtsbarkeit 37ff.

${ }^{71}$ Vgl. OsTERKAMP, Verfassungsgerichtsbarkeit 151ff.
} 
wurde nicht nur der Kreis der Antragsberechtigten um zwei Justizorgane erweitert, sondern auch die Abstraktheit des Verfassungskontrollverfahrens gemildert. Das von den obersten Gerichten angegriffene Gesetz musste für diese entscheidungsrelevant sein; kam das Verfassungsgericht zu dem Schluss, dass das entsprechende Gesetz verfassungswidrig sei, so sollte die Entscheidung nicht nur für die Zukunft gelten, sondern ausnahmsweise auf den sogenannten Anlassfall zurückwirken. ${ }^{72}$ Nach Ansicht eines amerikanischen Verfassungsrechtlers rückte das österreichische Modell damit ein Stück näher an das allgemeine richterliche Prüfungsrecht amerikanischen Zuschnitts, wodurch die „schlimmsten Mängel des Systems von 1920 ausgeglichen wurden ". ${ }^{73}$ Im Vergleich zur Tschechoslowakei waren die obersten Gerichte in Österreich jedenfalls weit stärker motiviert, von ihrer Kompetenz zur Verfassungskontrolle Gebrauch zu machen.

\section{Kontrolle der "Notverordnungspraxis"}

Angesichts dieser Abstraktheit und Objektivität der Verfassungskontrolle von Gesetzen lag der Schwerpunkt der Rechtsprechung des tschechoslowakischen Verfassungsgerichts auf der Kontrolle der vorläufigen Verfügungen des Ständigen Ausschusses, die es von Amts wegen untersuchte. Diese Verfügungen waren ein politisches Notinstrumentarium für Zeiten, in denen das Parlament nicht tagte - bei Neuwahlen, nach dessen Auflösung oder in den Parlamentsferien. Auch wenn diese Verfügungen von den Zeitgenossen oft als "Notverordnungen“ tituliert wurden, war der Ständige Ausschuss formell kein Organ der Exekutive, sondern setzte sich aus 24 Parlamentariern zusammen. Die vorläufigen

\footnotetext{
${ }^{72}$ Dies wurde indirekt aus der Verfassungsnovelle geschlossen und erst 1975 gesetzlich verankert. Kritisch KelSEN, Die Verfassung Österreichs 153; vgl. auch HALLER, Prüfung von Gesetzen 277ff.

${ }^{73}$ CAppelletti, Judicial Review 73f.
}

Verfügungen des Ständigen Ausschusses traten außer Kraft, wenn sie nicht nachträglich vom Parlament genehmigt wurden. Durch diese Anbindung an das Parlament wollte man eine ausgreifende Verordnungspraxis der Regierung verhindern, wie sie sich in der späten Habsburgermonarchie aufgrund des Art. 14 des Staatsgrundgesetzes über die Reichsvertretung herausgebildet hatte.

In der Verfassungswirklichkeit zeigte sich bald, dass die Gefahr einer politischen Machtverschiebung vom Parlament auf die Regierung durch die Institution des Ständigen Ausschusses keinesfalls gebannt war. Die staatliche Organisation des aus verschiedenen historischen und politischen Zusammenhängen herausgelösten, neu zusammengesetzten tschechoslowakischen Gemeinwesens erforderte in der Nachkriegszeit oft ein rasches Handeln. Die zum Teil wirtschaftsdirigistischen Verfügungen des Ständigen Ausschusses reagierten auf zeitgenössische Probleme wie Lebensmittel-, Wohnraumknappheit und Arbeitslosigkeit. Eine Verfügung vom Sommer 1920 ging erheblich weiter und regelte die Eingliederung der beiden landschaftlich reizvollen, aber auch wirtschaftlich wichtigen Gebiete um Weitra [Vitorazsko] und Feldsberg [Valčicko] im Süden von Böhmen und Mähren. Die Ratifizierung des Friedensvertrags von St. Germain war in die parlamentarische Sommerpause gefallen und die Inkorporation dieser ursprünglich zu Österreich gehörenden Gebiete lag nun in den Händen des Ständigen Ausschusses. ${ }^{74}$ Dieser stellte der Regierung eine Blankettermächtigung aus, „alle zur Erhaltung der Rechtsordnung und zur Einführung der ordentlichen Verwaltung und ordentlichen Gerichtsbarkeit erforderlichen Verfügungen $\mathrm{zu}$ treffen“ ${ }^{75}$ Diese Blankettermächtigung war nur eine unter vielen, welche der Regierung nicht

\footnotetext{
${ }^{74}$ OSTERKAMP, Verfassungsgerichtsbarkeit 94ff.

${ }^{75} \S 4$ Inkorporationsverfügung des Ständigen Ausschusses, Nr. 450/1920 Slg.
} 
nur vom Ständigen Ausschuss, sondern auch vom Parlament selbst zugestanden wurden. Das Verfassungsgericht machte sie zum Präzedenzfall gegen die ausufernde Verordnungsgewalt.

Das Verfassungsgericht war die erste öffentliche Institution, die die Ermächtigungspraxis von Parlament und Regierung in Frage stellte. In seinem ersten Urteil überhaupt, am 7. November 1922, sprach es sich gegen Ermächtigungsklauseln und gegen eine Verschiebung der gesetzgeberischen Gewalt auf die Exekutive aus. $^{76}$ Die verfassungsrechtliche Kompetenz zur Gesetzgebung sei kein „Recht" des Parlaments, das es auf andere Organe übertragen könne, sondern dessen Verfassungspflicht. Der in der Verfassung verankerte Gesetzesvorbehalt für Verordnungen werde ausgehöhlt, lege das Parlament nicht selbst die Leitsätze fest, nach der sich die Verordnungsgewalt $\mathrm{zu}$ richten habe. Angesichts der bereits etablierten politischen Praxis von Ermächtigungsklauseln kam dieses Urteil für viele überraschend. Die indirekte Bezugnahme des Gerichts auf das verfassungsrechtliche Demokratiemodell löste eine produktive rechtswissenschaftliche Debatte über das Verhältnis von Verfassung, Gesetz und Verordnung aus, an der sich auch ausländische Verfassungsrechtler wie Hans Kelsen, Léon Duguit und Maurice Hauriou beteiligten. ${ }^{77}$ In der Ministerialbürokratie stieß das Verbot von Ermächtigungsgesetzen auf eine verhaltene Resonanz. Spätestens infolge der Weltwirtschaftskrise setzte sich über das politische Instrumentarium von Ermächtigungsgesetzen und Notverordnungen die Machtverschiebung vom Parlament auf die Regierung fort. Nicht zuletzt aus Furcht, es kön-

\footnotetext{
${ }^{76}$ Das Urteil ist abgedruckt in deutscher Fassung bei ADLER, Gesetz und Verordnung $114 \mathrm{ff}$.

77 Deren Beiträge finden sich in der politischen Zeitschrift „Parlament“ aus dem Jahr 1923: KELSEN, Verhältnis Gesetz und Verordnung 392ff. sowie DUGUIT, HAURIOU, [ohne Titel] 391f. Zur Debatte in Rechtswissenschaft und Verwaltung vgl. im Übrigen OSTERKAMP, Verfassungsgerichtsbarkeit 111ff., $129 \mathrm{ff}$.
}

ne diese Ermächtigungsgesetze der dreißiger Jahre für verfassungswidrig erklären, ließ man das Verfassungsgericht zwischen 1931 und 1937 unbesetzt. Erst im Jahr 1939, bereits unter den Bedingungen des „Protektorats", erklärte das Verfassungsgericht eines dieser Ermächtigungsgesetze für verfassungswidrig und schlug damit den Bogen zu seiner ersten Entscheidung.

\section{Dritter Teil: Fazit}

Das Modell der tschechoslowakischen Verfassungsgerichtsbarkeit orientierte sich ausschließlich am Vorrang der Verfassung. Die übrigen Faktoren - Föderalismusidee und Nationalitätenproblem -, die in der Habsburgermonarchie ideengeschichtlich zur Entwicklung der konzentrierten Verfassungsgerichtsbarkeit beigetragen hatten, spiegelten sich in dieser Konstruktion nicht wieder.

Dies lag einerseits am zentralistischen Aufbau des neuen Staates, dessen Verfassung gegenüber der Vielfalt der Tschechoslowakei immun blieb. Die erheblichen historischen, wirtschaftlichen, sozialen und nationalen Unterschiede zwischen den Regionen Böhmen, Mähren, MährischSchlesien, der Slowakei und der Karpathenukraine wurden durch die Idee des tschechoslowakischen Einheitsstaats verfassungsrechtlich überspielt. Mit Blick auf das Nationalitätenproblem beschränkte sich die tschechoslowakische Verfassung andererseits auf einen umfangreichen Katalog nationaler Minderheitenrechte. Ältere Ideen einer Nationalitätengerichtsbarkeit griffen die Verfassungsväter nicht auf. Auf die Konflikte zwischen den verschiedenen Regionen und zwischen den miteinander streitenden Nationalitäten konnte das tschechoslowakische Verfassungsgericht daher nicht integrativ einwirken. Die rasche Wiederkehr der aus der Habsburgermonarchie bekannten Forderungen nach Föderalisierung und einem Nationalitätengerichtshof zeigt jedoch die 
anhaltende Attraktivität einer zentralen Gerichtsbarkeit als Lösung politischer Konflikte. ${ }^{78}$ In ideengeschichtlicher Hinsicht ist die Loslösung der Verfassungsgerichtsbarkeit von Föderalismus und Minderheitenproblem zugleich eine Stärke des tschechoslowakischen Modells. Es eröffnet sich dadurch der Blick auf die allgemeinen Anforderungen der Verfassungsgerichtsbarkeit in der modernen Verfassungsdemokratie, unabhängig davon, ob es sich um einen Einheits- oder Föderalstaat, einen National- oder Nationalitätenstaat handelt. In einem parlamentarischen Regierungssystem, das dem Gesetzgeber die primäre Entscheidungsgewalt überträgt, gleichzeitig dessen Machtausübung jedoch an bestimmte Leitvorgaben der Verfassung bindet, kann Verfassungsgerichtsbarkeit einen wirksamen Schutz vor VerfassungsverstöBen bieten. Das Funktionieren dieses Kontrollmechanismus hängt vom stimmigen Zusammenspiel der anderen Faktoren des gewaltenteiligen Systems ab. Im tschechoslowakischen Fall wirkte sich etwa das von Parteistaatlichkeit überformte Zweikammersystem und eine politisch abhängige Wahlgerichtsbarkeit negativ aus. Als weiteres hemmendes, dem tschechoslowakischen Modell der Verfassungsgerichtsbarkeit selbst innewohnendes Merkmal ist die Abstraktheit der Verfassungskontrolle zu nennen. Nach dem Willen der Verfassungsgeber sollte diese dazu dienen, das Verfahren vor dem Verfassungsgericht von partikularen Interessen zu entlasten. Es kam ihnen auf die objektive Sicherung der Rechtsordnung an. Damit wurde jedoch letztlich entscheidenden Motivationsfaktoren die produktive Kraft genommen und ein verfassungsgerichtlicher Alltag verhindert. Dem Verfassungsgericht fehlte der Rückhalt in den

\footnotetext{
${ }^{78}$ Die Föderalisierung der Tschechoslowakei und der damit einhergehende Ausbau der Kompetenzen des tschechoslowakischen Verfassungsgerichts vom Dezember 1938 entfalteten allerdings keinerlei Wirkung mehr.
}

entscheidenden politischen Institutionen. Die pragmatischere Lösung in Österreich mit einer Rückwirkung auf den "Anlassfall“ erwies sich demgegenüber als erfolgreicher. Für das kontinentaleuropäische Modell der „konzentrierten Verfassungsgerichtsbarkeit" sind beide Erfahrungen von verfassungshistorischer Bedeutung. 


\section{Literatur:}

Ludwig ADAmOvich, Der Kremsierer Entwurf und die österreichische Bundesverfassung, in: Zeitschrift für öffentliches Recht (Wien 1927) 561-571.

Ludwig AdAmovich, Die Prüfung der Gesetze und Verordnungen durch den österreichischen Verfassungsgerichtshof (Leipzig-Wien 1923).

Franz ADLER, Das parlamentarische Regierungssystem in der tschechoslowakischen Verfassung, in: Prager Juristische Zeitschrift (Prag 1930) 297-306.

Franz ADLER, Gesetz und Verordnung, in: Prager Juristische Zeitschrift 2 (1923) $114 \mathrm{ff}$.

Franz ADLER, Verfassung und Richteramt. Ein Beitrag zur Frage des richterlichen Prüfungsrechtes, in: Zeitschrift für öffentliches Recht (Wien 1931), $103 \mathrm{ff}$.

Josef BLAHOŽ, Soudní kontrola ústavnosti. Srovnávací pohled, in: Georg BRUNNER, Mahulena HofFMANN, Pavel HOLLÄNDER (Hgg.), Verfassungsgerichtsbarkeit in der Tschechischen Republik (BadenBaden 2001) 13ff., 53ff

Eva BROKLOVÁ, První československá ústava. Diskuse v ústavním výboru v lednu a únoru 1920 (Praha 1992).

Georg BRUnNer, Mahulena HoffMAnN, Pavel HoLLÄNDER, Verfassungsgerichtsbarkeit in der Tschechischen Republik (Baden-Baden 2001).

Winfried BRUGGER, Kampf um die Verfassungsgerichtsbarkeit. 200 Jahre Marbury v. Madison, in: Juristische Schulung (München 2003) 320-325.

Mauro CAPPELLETTI, Judicial Review in the Contemporary World (Indianapolis 1971).

Johann Gustav DROYSEN, Die Verhandlungen des Verfassungsausschusses der deutschen Nationalversammlung, Bd. 1 (Leipzig 1849).

L. Duguit, M. Hauriou, [ohne Titel], in: Parlament (1923) 391f.

József von EÖTVÖs, Der Einfluß der herrschenden Ideen des 19. Jahrhunderts auf den Staat, Bd. 2 (Leipzig 1854).

Hans Joachim FALLER, Die Verfassungsgerichtsbarkeit in der Frankfurter Reichverfassung vom 28. März 1849, in: DERS., u.a. (Hgg.), Menschenwürde und freiheitliche Rechtsordnung (Tübingen 1974) 827866.

Hans Joachim FALLER, Zur Entwicklung der nationalen Verfassungsgerichte in Europa, in: Europäische Grundrechte Zeitschrift (1986) 42-60.

Otakar FLANDERKA, Le contrôle de la constitutionalité des lois en Tchécoslovaquie (Paris 1926).
Zaccaria GIACOMETTI, Die Verfassungsgerichtsbarkeit des Schweizerischen Bundesgerichtes (Die staatsrechtliche Beschwerde) (Zürich 1933).

Andreas GotTSMANN, Der Reichstag von Kremsier und die Regierung Schwarzenberg (Wien 1995).

Herbert Haller, Die Prüfung von Gesetzen. Ein Beitrag zur verfassungsrechtlichen Normenkontrolle (Wien 1979).

Paul HARTMANN, Die Überprüfung der Gesetze nach tschechoslowakischem Rechte (Prag 1928).

Joseph Alexander HeLFERT, Aufzeichnungen und Erinnerungen aus jungen Jahren (Wien 1904).

Kurt Heller, Der Verfassungsgerichtshof. Die Entwicklung der Verfassungsgerichtsbarkeit in Österreich von den Anfängen bis zur Gegenwart (Wien 2010).

Jiři HoETZEL, Ústava, in: E. CHALuPNÝ (Hg.), Slovník národohospodářský, sociální a politický III, 479.

Karl HugelmanN, Das österreichische Reichsgericht, in: Zeitschrift für öffentliches Recht (Wien 1925), 458-538.

Walter JELLINEK, Der Schutz des öffentlichen Rechts durch ordentliche und durch Verwaltungsgerichte, in: Veröffentlichungen der Vereinigung Deutscher Staatsrechtslehrer (Berlin-Leipzig 1925) 880.

Georg JELLINEK, Ein Verfassungsgerichtshof für Österreich (Wien 1885).

Hans KeLSEN, Das Verhältnis von Gesetz und Verordnung nach der čechoslowakischen Verfassungsurkunde, in: Parlament (1923) 392-396.

Hans KELSEN, Die Verfassung Österreichs, in: JöR 1930, 130-185.

Jens KERSTEN, Mehrheit und Minderheit im Minoritätenstaat, in: Der Staat (2001), 221ff.

Jaroslav KREJČÍ, Die Prüfung der Verfassungsmäßigkeit von Gesetzen in der čechoslovakischen Republik, in: Zeitschrift für Ostrecht 1929, 38-47.

Jaroslav KREJČí, Principy soudcovského zkoumání zákonů v právu československém (Praha 1932).

Jörg-Detlef KüHNE, Die Reichsverfassung der Paulskirche. Vorbild und Verwirklichung im späteren Rechtsleben (Neuwied 21998).

Jan KYSELA, Dvoukomorové systémy. Teorie, historie a srovnání dvoukomorových parlamentů (Praha 2004).

Tomáš LANGÁŠEK, Ústavní soud Československé republiky a jeho osudy v letech 1920-1948 (Plzeň 2011).

Karl von LEMAYER, Rechtsschutz im öffentlichen Rechte, in: Ernst MischleR, Josef Ulbrich (Hgg.), 
Österreichisches Staatswörterbuch, Bd. 4 (Wien 21907) 17-53.

Stefan MALFÉR, Kremsier in der österreichischen Verfassungsdiskussion um 1900, in: Der Reichstag von Kremsier 1848-1849 und die Tradition des Parlamentarismus in Mitteleuropa (Kremsier 1998) 65-76.

René MARCIC, Verfassung und Verfassungsgericht (Wien 1963).

Erwin MELICHAR, Die Verfassungsgerichtsbarkeit in Österreich, in: Max Planck-Institut für ausländisches öffentliches Recht und Völkerrecht (Hg.), Verfassungsgerichtsbarkeit in der Gegenwart, $439 \mathrm{ff}$.

Friedrich MERZBACHER, Austrägalinstanz, in: Handwörterbuch zur deutschen Rechtsgeschichte, Bd. 1 (Aachen-Haussuchung ${ }^{1} 1971$ ) 273-274.

Alfred J. NoLL, Georg Jellineks Forderung nach einem Verfassungsgerichtshof für Österreich, in: Stanley L. Paulson, Martin Schulte (Hgg.), Georg Jellinek. Beiträge zu Leben und Werk (Tübingen 2000) 261-276.

A. Nostitz, Entwurf einer Verfassungs-Urkunde für das Kaiserthum Oesterreich (Karlsbad o.J. [1848]).

Theo ÖHLINGER, Die Entstehung und Entfaltung des österreichischen Modells der Verfassungsgerichtsbarkeit, in: Bernd-Christian FUNK (Hg.), Der Rechtstaat vor neuen Herausforderungen. Festschrift für Ludwig Adamovich (Wien 2002) 581600 .

Jana OSTERKAMP, Verfassungsgerichtsbarkeit in der Tschechoslowakei 1920-1939. VerfassungsideeDemokratieverständnis-Nationalitätenproblem (Frankfurt am Main 2009).

Stanley PAULSON, Constitutional Review in the United States and Austria. Notes on the Beginnings, in: Ratio juris 16(2003) 223-239.

Karl RENNER, Das Selbstbestimmungsrecht der Nationen in besondere Anwendung auf Österreich, Erster Teil: Staat und Nation (Wien 1918).

Karl RENNER, Der Kampf der österreichischen Nationen um den Staat (Wien-Leipzig 1902).

Bohuš RIEGER (Hg.), Františka Palackého Spisy drobné Bd. 1 (Praha 1898).

Bohuš RIEGER, Ústava Rakouska dle Fr. Palackého v 1. 1848-49, in: Památník na oslavu stých narozenin Františka Palackého (Praha 1898) 602ff.

Ulrich SCHEUNER, Die Überlieferung der deutschen Staatsgerichtsbarkeit im 19. und 20. Jahrhundert, in: Christian STARCK (Hg.), Bundesverfassungsgericht und Grundgesetz (Tübingen 1976) 1-62.

Vit SCHORM, La cour constitutionnelle de la république tchécoslovaque d'entre deux guerres: Une première oubliée, in: Annuaire International de Justice Constitutionnelle (Marseille 2001) 11-28.

Vladimír SLÁDEČEK, Ústavní soudnictví (Praha 2003).

Anton SPRINGER, Protokolle des Verfassungsausschusses im österreichischen Reichstage. 18481849 (Leipzig 1885).

Christian Starck, Albrecht Weber (Hgg.), Verfassungsgerichtsbarkeit in Westeuropa, Bd 1 (BadenBaden 1986).

Gerald STOURZH, Die Gleichberechtigung der Nationalitäten in der Verfassung und Verwaltung Österreichs 1848-1918 (Wien 1985).

Gerald STOURZH, Georg Jellinek. Die altösterreichische Verfassungswirklichkeit und das Problem der Verfassungsgerichtsbarkeit, in: Stanley L. PAULSON, Martin Schulte (Hgg.), Georg Jellinek. Beiträge zu Leben und Werk (= Beiträge zur Rechtsgeschichte des 20. Jahrhunderts 27) (Tübingen 2000) 247-260.

Gerald STOURZH, Vom Widerstandsrecht zur Verfassungsgerichtsbarkeit. Zum Problem der Verfassungswidrigkeit im 18. Jahrhundert (Graz 1974).

Michel Troper, Kelsen und die Kontrolle der Verfassungsmäßigkeit, in: Agostino CARRINO, Günther WINKLER (Hgg.), Rechtserfahrung und Reine Rechtslehre (Wien 1995) 15-39.

Karl Theodor WELCKER, Schiedsgerichte, in: DERS., Karl von ROTTECK (Hgg.), Staats-Lexikon oder Encyklopädie der Staatswissenschaften, Bd. 14 (Altona 1843) 253-282.

Ewald WIEDERIN, Bundesrecht und Landesrecht (=Forschungen aus Staat und Recht 11) (Wien 1995).

Dieter WYDUCKEL, Georg Jellineks Beitrag zur Entwicklung der Verfassungsgerichtsbarkeit, in: Stanley L. PAulson, Martin Schulte (Hgg.), Georg Jellinek. Beiträge zu Leben und Werk (= Beiträge zur Rechtsgeschichte des 20. Jahrhunderts 27) (Tübingen 2000) 277-298.

\section{Abkürzungen:}

NA Národní archiv

ÚS Ústavní soud 